\title{
On Topology of Sensor Networks Deployed for Multi-Target Tracking
}

Ye Zhu

Cleveland State University, y.zhu61@csuohio.edu

A. Vikram

Huirong Fu

Oakland University, fu@oakland.edu

Follow this and additional works at: https://engagedscholarship.csuohio.edu/enece_facpub How does access to this work benefit you? Let us know!

\section{Publisher's Statement}

(C) 2014 IEEE. Personal use of this material is permitted. Permission from IEEE must be obtained for all other uses, in any current or future media, including reprinting/republishing this material for advertising or promotional purposes, creating new collective works, for resale or redistribution to servers or lists, or reuse of any copyrighted component of this work in other works.

\section{Repository Citation}

Zhu, Ye; Vikram, A.; and Fu, Huirong, "On Topology of Sensor Networks Deployed for Multi-Target Tracking" (2014). Electrical Engineering \& Computer Science Faculty Publications. 301.

https://engagedscholarship.csuohio.edu/enece_facpub/301

This Article is brought to you for free and open access by the Electrical Engineering \& Computer Science Department at EngagedScholarship@CSU. It has been accepted for inclusion in Electrical Engineering \& Computer Science Faculty Publications by an authorized administrator of EngagedScholarship@CSU. For more information, please contact library.es@csuohio.edu. 


\title{
On Topology of Sensor Networks Deployed for Multitarget Tracking
}

\author{
Ye Zhu, Member, IEEE, Anil Vikram, and Huirong Fu, Member, IEEE
}

\begin{abstract}
In this paper, we study topologies of sensor networks deployed for tracking multiple targets. Tracking multiple moving targets is a challenging problem. Most of the previously proposed tracking algorithms simplify the problem by assuming access to the signal from an individual target for tracking. Recently, tracking algorithms based on blind source separation (BSS), a statistical signal-processing technique widely used to recover individual signals from mixtures of signals, have been proposed. BSS-based tracking algorithms are proven to be effective in tracking multiple indistinguishable targets. The topology of a wireless sensor network deployed for tracking with BSS-based algorithms is critical to tracking performance because the topology affects separation performance, and the topology determines accuracy and precision of estimation on the paths taken by targets. We propose cluster topologies for BSS-based tracking algorithms. Guidelines on parameter selection for proposed topologies are given in this paper. We evaluate the proposed cluster topologies with extensive experiments. Our experiments show that the proposed topologies can significantly improve both the accuracy and the precision of BSS-based tracking algorithms.
\end{abstract}

Index Terms-Blind source separation (BSS), cluster, topology, tracking.

\section{INTRODUCTION}

$\mathbf{I}$ $\mathrm{N}$ THIS paper, we investigate topologies of sensor networks deployed for tracking multiple targets. We focus our attention on tracking algorithms based on blind source separation (BSS) algorithms.

Tracking moving targets with wireless sensors is one of the prominent applications of wireless sensor networks. Sensors, also called "smart dust" [1], are small devices known for their simplicity and low cost. Using a network of sensors with wireless communication capability enables both cost- and performance-effective approaches to track targets, due to the availability of a large amount of data collected by sensors for tracking targets. Depending on the applications, sensors with different sensing modalities such as acoustic, seismic, infrared, radio, and magnetic can be deployed for tracking different types of targets.
In general, data collected by sensors are aggregate data. In other words, signals received by sensors are, generally, mixtures of signals from individual targets. For example, an acoustic sensor in a field of interest may receive sound signals from more than one target. Obviously, tracking targets based on mixture signals cannot be accurate when interference from targets other than the one of interest is not negligible. Without loss of generality, in the rest of this paper we use the term individual signal to mean the signal transmitted from or caused by individual targets. We use the term aggregate signal to mean the signal received by sensors, i.e., data collected by sensors. One aggregate signal received by a sensor is essentially a mixture of the individual signals from targets.

The fact that signals collected by sensors networks are aggregate signals poses a big challenge to target-tracking solutions. Most previous research simplifies the general multitarget tracking problem and the simplified problems have been well studied.

- Single-target case: In this case, it is assumed that only one target exists in a field of interest. Thus, signals received by sensors are essentially individual signals.

- Negligible interference case: Some research assumes that the interference from targets other than the one of interest is negligible. The assumption is legitimate only for applications in which the signal from a target dramatically attenuates when the distance between the target and the sensor increases.

- Distinguishable target case: Sensors can distinguish targets by tags embedded in signals or by having different targets to send signals using different channels such as using different frequency bands.

All these special cases assume that tracking algorithms can have access to individual signals.

Singh et al. [2] proposed a general approach to track multiple targets indistinguishable by sensors. The approach is based on binary proximity sensors that can only report whether there are targets in a sensing area. The approach is simple and is robust to interference from other targets, with the cost of limitation that it is only applicable to tracking targets in smooth paths [2].

We propose tracking algorithms to solve the general multitarget tracking problem in [3]. The algorithms are based on BSS, a methodology from statistical signal processing to recover unobserved "source" signals from a set of observed mixtures of the signals. BSS models were originally defined to solve a cocktail party problem: The BSS algorithms can extract one person's voice signal given the mixtures of voices in a cocktail party. BSS algorithms solve the problem based on the independence between voices from different persons. Similarly, 
in the target-tracking problem, it is generally safe to assume that individual signals from different targets are independent. Thus, we can use BSS algorithms to recover individual signals from aggregate signals collected by sensors. For the cases in which individual signals are dependent, BSS algorithms based on timing structures [4] of individual signals can be used. Interested readers may refer to [5] for an evaluation on the performance of BSS in separating sensor signals collected from a distributed network.

In this paper, we focus on topologies of a sensor network to improve tracking performance. The topology of a sensor network deployed for tracking is critical to tracking performance. First, the topology affects separation performance. For better separation performance, sensors should be clustered so that there are more sensors than targets within the sensing ranges of these sensors and, in general, better separation performance leads to better tracking performance. When the number of targets is larger than the number of the sensors, it means that the number of source signals is larger than the number of observed mixtures. Usually, the separation problems of having more source signals than observed mixtures are called overcomplete bases problems [6], which are considered to be harder problems, and applying generic BSS algorithms may not fully separate the source signals. Second, the number of sensors that can "hear" a target of interest determines how accurate and precise a BSS-based tracking algorithm can estimate the path taken by the target.

We propose cluster-based topologies for better tracking performance. Our contributions in this paper can be summarized as follows.

- We list necessary requirements for candidate topologies.

- We propose the use of cluster-based topologies to improve tracking performance. Guidelines on selected parameters of proposed cluster topologies are given in this paper. We evaluate proposed topologies with extensive experiments.

- We propose metrics to evaluate the performance of the proposed topologies using target-tracking algorithms. The metrics originate from the general metrics used to evaluate performance of an estimator in statistics since, essentially, target tracking algorithms estimate the paths based on data collected from sensor networks.

The rest of this paper is organized as follows. Section II reviews related work. Section III formally defines the problem. We describe the proposed topologies in Section IV. We evaluate the proposed topologies under various settings in Section V. We conclude this paper in Section VII.

\section{RELATED WORK}

Tracking multiple targets in a field is a challenging problem compared with tracking a single target. Some research on tracking targets with wireless sensor networks is based on binary proximity sensors, which can only report whether there are targets within sensing areas. The initial work [7] on binary proximity sensors focuses on tracking a single target. Singh et al. [2] extended the approach to track multiple indistinguishable targets by applying particle filtering algorithms. Approaches based on binary proximity sensors have two ob- vious advantages: the sensors are very simple since they only report binary information and the approaches are robust since interference from other targets is essentially filtered out by an equivalent low-passed filter [7]. The cost of using these simple devices is a loss of information that is helpful to accurately track targets due to the filtering effect. Hence, approaches based on binary proximity sensors cannot track target in a path with high-frequency variations [7]. Since the BSS-based tracking algorithms are able to recover individual signals for tracking, the BSS-based algorithms can track targets following paths with high-frequency variations. Interested readers may refer to [3] for more comparison and experiment results.

A research problem related to the target tracking is target identification or target classification. Various advanced techniques have been applied to solve the problem. Friedlander and Phoha [8] proposed statistical techniques based on principal component analysis for target identification with knowledge on target position, time, target velocity, and a set of target attributes collected from sensors. In [9], the authors proposed wavelet-based algorithms for target classification. In this paper, we propose algorithms based on the BSS algorithms for target tracking.

Topologies of sensor networks have been studied for different purposes.

- Coverage: In [10], Bai et al. proposed optimal deployment to achieve full coverage. Different patterns such as polygon, diamond, and square are proposed for sensor deployment topologies. To ensure full coverage and connectivity, decentralized density control algorithms were proposed in [11]. In [12], Kershner proposed a triangular pattern, which is optimal in terms of number of circles needed to entirely cover a given area.

- Localization: Zou and Chakrabarty [13] proposed a sensor deployment for target localization based on the virtual force algorithm, which attempts to maximize the sensor field coverage. Chan et al. [14] proposed a localization approach without requiring any ranging or positioning equipment.

- Communication cost: Kasetkasem and Varsheny [15] studied the problem of communication structure planning in sensor detection systems, based on communication constraints and generalized cost formulation.

In this paper, we focus on topologies of sensor networks deployed for tracking.

Clustering has been proposed for wireless sensor networks. Examples are as follows. Alaybeyoglu et al. [16] evaluated the cluster-based target tracking protocols for wireless sensor networks and Bhatti et al. [17] studied clustering and fault tolerance for target tracking. Our work is unique in two aspects. First, the related work (including the two papers listed above) focuses on logical clustering, in which the sensors are deployed either randomly or as grid. Then, the sensor nodes are grouped into a cluster and a cluster head is selected among the sensor nodes for different purposes, e.g., effective communication, power saving, or tracking. Our paper focuses on physical clustering, in which the sensor nodes are physically deployed as clusters at nearby physical locations. Second, the 


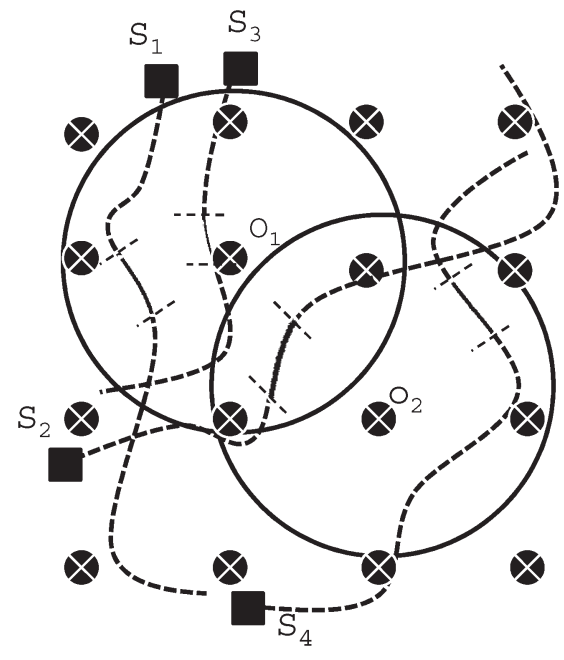

Fig. 1. System model. Black circles with a cross in the middle represent sensors, black squares represent targets, dashed lines represent the moving paths of the targets, and circles with solid lines represent the sensing ranges of the sensors.

related work (including the two papers listed above) focuses on tracking a single target, whereas our approach focuses on tracking multiple targets.

\section{Problem Statement}

Here, we begin with the system model and the goal of this paper and then proceed with the description on application of BSS algorithms in target tracking. We finish this section with the requirements on candidate topologies.

\section{A. System Model and Goal}

A general model of tracking targets using wireless sensor networks is shown in Fig. 1. Wireless sensors are deployed in certain topology in a field of interest. In general, a wireless sensor receives signals from multiple targets. For example, supposing that acoustic sensors are deployed in Fig. 1, Sensor $O_{1}$ can receive audio signals from Targets $S_{1}, S_{2}$, and $S_{3}$ at the same time. Thus, the signal received by Sensor $O_{1}$ is an aggregate signal, i.e., a combination of individual signals transmitted by Targets $S_{1}-S_{3}$.

The goal of this paper is to improve tracking performance for tracking multiple targets with BSS algorithms. In general, tracking algorithms output an estimated area for a path segment, as shown in Fig. 2. We use mean and standard deviation of error distance to measure tracking performance in this paper. The error distance is defined as the nearest distance between a specific point in the estimated area to the actual path taken by a target, as shown in Fig. 2. The mean and the standard deviation of the error distance are calculated based on all the points in the estimated area. The mean and the standard deviation of the error distance measure the accuracy and the precision of the tracking algorithm, respectively. If we cast the evaluation of the estimation algorithm in terms of evaluating a statistical estimator, the accuracy corresponds to the bias of the estimator, and the precision corresponds to the variance of the estimator.

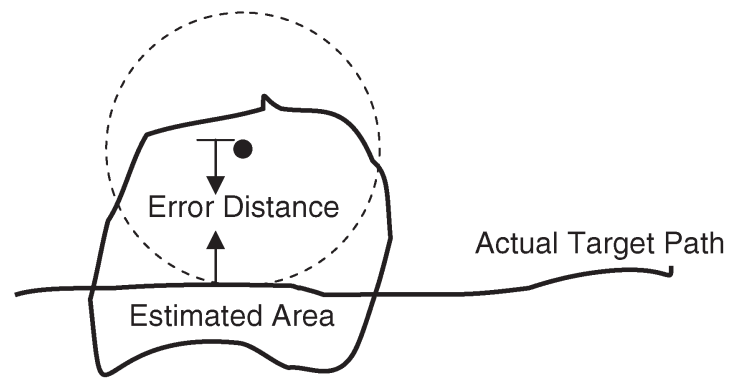

Fig. 2. Error distance. The solid dot represents one point in the estimated area. The circle in the dashed line shows that the error distance is the nearest distance between the point and the actual target path.

\section{B. Application of BSS Algorithms in Tracking Targets}

In this paper, we are particularly interested in the topologies of sensor networks deployed for multitarget tracking with tracking algorithms based on the BSS technique because BSS-based tracking algorithms can solve the general multitarget tracking problem even when the targets are moving along paths of highfrequency variation [3]. We introduce BSS and the rationale of applying BSS to the multitarget tracking problem below.

1) BSS: BSS is a methodology used in statistical signal processing to recover unobserved source signals from a set of observed mixtures of the signals. The separation is called blind to emphasize that the source signals are not observed and that the mixture is a black box to the observer. While no knowledge is available about the mixture, in many cases, it can be safely assumed that source signals are independent. In its simplest form [18], the BSS model assumes $n$ independent signals $S_{1}(t), \ldots, S_{n}(t)$ and $n$ observations of mixture $O_{1}(t), \ldots, O_{n}(t)$, where $t$ denotes the time ${ }^{1}$ and $O_{i}(t)=$ $\sum_{j=1}^{n} a_{i j} S_{j}(t)$. The goal of BSS is to reconstruct the source signals $S_{j}(t)$ using only the observed data $O_{i}(t)$, the assumption of independence among the signals $S_{j}(t)$. Given the observations $O_{i}(t)$, BSS techniques estimate the signals $S_{j}(t)$ by maximizing the independence between the estimated signals. A very nice introduction to the statistical principles behind BSS is given in [18]. The common methods employed in BSS are minimization of mutual information [19], [20], maximization of non-Gaussianity [21], [22], and maximization of likelihood [23], [24]. Timing-structure-based algorithms [4] can be used to recover source signals when source signals are dependent.

2) Recover Individual Signals for Target Tracking With BSS Algorithms: In our tracking approach, BSS algorithms are used to recover individual signals, i.e., source signals, as described above, from aggregate signals, i.e., observations, as described above. Supposing that acoustic sensors are deployed in the field shown in Fig. 1, Sensor $O_{1}$ can receive audio signals from Targets $S_{1}, S_{2}$, and $S_{3}$; and Sensor $O_{2}$ can receive audio signals from Targets $S_{2}$ and $S_{4}$. If we represent the signal received by Sensor $O_{i}$ as $O_{i}(t)$ and the signal from Target $S_{i}$ as $S_{i}(t)$, we can have the following two equations: $O_{1}(t)=$ $S_{1}(t)+S_{2}(t)+S_{3}(t)$, and $O_{2}(t)=S_{2}(t)+S_{4}(t)$. In general,

${ }^{1}$ Essentially, the signals are functions of time $t$. 
for $m$ neighboring sensors and $n$ targets, we can rewrite these equations in vector-matrix notation, i.e.,

$$
\left(\begin{array}{c}
O_{1}(t) \\
O_{2}(t) \\
\vdots \\
O_{m}(t)
\end{array}\right)=\mathbf{A}_{m \times n}\left(\begin{array}{c}
S_{1}(t) \\
S_{2}(t) \\
\vdots \\
S_{n}(t)
\end{array}\right)
$$

where $\mathbf{A}_{m \times n}$ is called the mixing matrix in the BSS literature. Since the individual signals are independent from each other-they come from different targets - we can use any of the algorithms mentioned in Section III-B1 to recover individual signals $S_{1}(t), \ldots, S_{n}(t)$. Given recovered individual signals, BSS-based tracking algorithms can use tracking techniques for individual signals such as overlapping sensing ranges of sensors that can receive similar recovered individual signals. An example tracking algorithm based on the overlapping technique is described in [25]. A common step in BSS-based tracking algorithms is to group $m$ neighboring sensors and apply BSS algorithms to recover individual signals $S_{1}(t), S_{2}(t), \ldots, S_{n}(t)$ from aggregated signals received by these $m$ sensors, i.e., $O_{1}(t), O_{2}(t), \ldots, O_{m}(t)$ in $(1)$.

\section{Requirements on Candidate Topologies}

We focus on the topologies of low-density sensor networks simply because the effect of the topologies on tracking performance is negligible for high-density sensor networks. In this paper, we assume candidate topologies that should satisfy the following requirements.

- Planned deployment: The deployment used in tracking targets can be classified into two categories: random deployment [26] and planned deployment [26], [27]. In random deployment, sensors are randomly distributed over the field. We eliminate random deployment from consideration because, for low-density sensor networks, the tracking performance of random deployment is usually worse than the tracking performance of planned deployment.

- Full coverage: In planned deployment, we focus on topologies enabling sensors to cover the whole field of interest. This requirement is particularly important for low-density sensor networks to prevent targets disappearing from tracking. One of the reasons for eliminating random deployment from consideration is its possibility of incomplete coverage for low-density sensor networks.

- Symmetrical topology: In this paper, we only consider symmetrical topologies. Symmetry is desirable since target motion direction should be unconstrained. Symmetrical topologies can ensure that tracking performance is direction independent.

\section{TOPOLOGY OF WIRELESS SENSOR NETWORKS FOR TRACKING}

Here, we describe the topologies proposed for BSS-based tracking algorithms. We start this section with the rationales behind the proposed topologies.

\section{A. Rationales Behind the Cluster Topologies}

The key step in BSS-based tracking algorithms is to apply BSS algorithms to recover individual signals from aggregate signals so that tracking algorithms can have access to individual signals. Obviously, the performance of separating out individual signals largely dictates overall tracking performance.

As described in Section III-B, the separation requires that a group of sensors receive different mixtures of individual signals from targets. In the context of target tracking, successful separation requires the following. First, the number of aggregate signals in one separation should be no less than the number of individual signals. In other words, the number of sensors involved in one separation should be no less than the number of targets involved in the separation because most BSS algorithms require the number of observations to be larger than or equal to the number of independent signals. Advanced BSS algorithms [28], [29] targeting the overcomplete base problem, in which the number of observations is less than the number of independent signals, usually require some assumptions on the individual signals. Second, the underlying individual signals received by two sensors involved in one separation should be similar to each other. Based on the rationales, we propose cluster topologies for tracking with BSS algorithms.

Before introducing the proposed topologies, we would like to use our preliminary experiments to show the topology impact on the separation performance.

\section{B. Topology Impact on Separation Performance}

To investigate the effect of the topology on separation performance, we did a series of initial experiments with random topologies.

In these initial experiments, 700 sensors are randomly distributed in a field of size $1.6 \mathrm{~km} \times 1.6 \mathrm{~km}$. To remove boundary issues, ${ }^{2}$ a total of 15 targets is restricted to move in the field center of size $1 \mathrm{~km} \times 1 \mathrm{~km}$. The sensing range of each sensor is $250 \mathrm{~m}$.

In the initial experiments, we assume that each target is moving and emitting sound signals. The sound signals used in the initial experiments are downloaded from the website of the Florida Museum of Natural History, Gainesville, FL, USA [30]. The aggregate sound signals received by the sensors are simulated by attenuating the sound signals from individual targets according to the atmospheric sound absorption model [31] and then mixing the attenuated individual signals. The BSS-based tracking algorithms recover the individual signals from the aggregate signals with BSS algorithms. The separation performance with different topologies can be evaluated by comparing recovered individual signals with the original individual signals from targets. A correlation-based metric denoted by $P_{\text {sep }}$ is used in our experiments to measure the separation performance. It is calculated by taking the absolute value of the correlation between one original individual signal and the corresponding recovered signal. We use the absolute value because one recovered signal may be of different sign in

\footnotetext{
${ }^{2}$ The boundary issues in this paper mean that targets may not be covered by sensors of enough density in one or more directions when targets are on an edge or a corner of the field covered by the sensors. Thus, we restrict the targets to move within the center of the field
} 


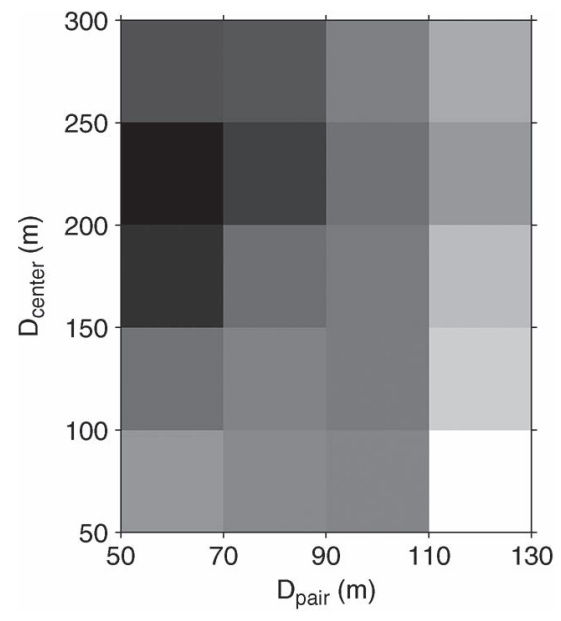

Fig. 3. Effect of topology on separation.

comparison with the corresponding original individual signal. The metric $P_{\text {sep }}$ is within the range $[0,1]$.

Two topology-related metrics are used in our analysis. We represent the first metric as $D_{\text {pair. }}$. It measures the average distance between each pair of sensors in a sensor group. The second metric, denoted by $D_{\text {center }}$, measures the average distance between a target of interest and the center of the sensor group of interest when the target is moving. Essentially, the metric $D_{\text {pair }}$ measures the clustering degree of neighboring sensors, and the metric $D_{\text {center }}$ measures the distance between a target and a cluster of sensors.

Fig. 3 shows the separation performance of these initial experiments. We present the separation performance visually as a data image in Fig. 3 - a grid with the metrics $D_{\text {pair }}$ and $D_{\text {center }}$ on the $x$ - and $y$-axes, respectively. The gray level of each pixel in the data image represents the separation performance $P_{\text {sep }}$ : A darker pixel indicates better separation performance, i.e., a larger value of $P_{\text {sep. }}$. From Fig. 3, we can observe the following. First, the separation performance is better when $D_{\text {pair }}$ is smaller. In other words, the separation performance is better for sensor groups with sensors closer to each other, i.e., clustered together. Second, the best separation performance is achieved when the target is away from one sensor group but still within the sensing range of the sensor group.

These two observations are because of signal attenuation, a natural consequence of signal transmission over long distances. Attenuation is a function of transmission distance. For moving targets, the distance between the target and the sensors changes over time. Thus, the attenuation is essentially a function of time. The attenuation functions even for two neighboring sensors are different. Thus, two neighboring sensors in a sensor group may receive different signals from a target.

The difference causes noise in separation. Obviously, when sensors are closer to each other and the target of interest is away from these sensors, the difference in attenuation functions of these sensors is relatively smaller. In turn, less noise is generated in separation, and better separation performance can be achieved.

In summary, our initial experiments indicate that topologies of clustered sensors are more suitable for BSS-based tracking algorithms since these topologies can lead to better separation performance.

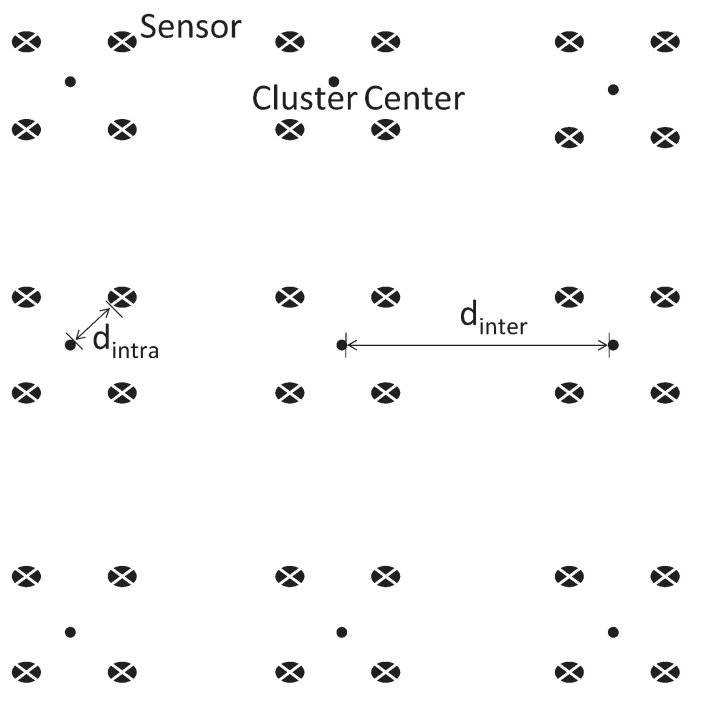

Fig. 4. Example of cluster topology.

\section{Proposed Topologies}

Based on the observations made in our initial experiments, we propose the cluster topologies for BSS-based tracking algorithms. The cluster topologies are general: any regular and symmetric topologies can be regarded as special cases of the cluster topologies. For example, the grid topology can be formed with only one node in each cluster. Our experiments indicate that better separation performance can be achieved when sensors are clustered together.

In the proposed topologies, as shown in Fig. 4, sensors are placed into clusters, and sensor clusters are evenly distributed in a field. The proposed cluster topologies have four parameters.

- In-cluster arrangement: Within each cluster, sensors are arranged in regular patterns. Possible choices are any polygon-based patterns such as well-known triangle lattice pattern, square pattern, pentagon pattern, and hexagon pattern.

- Number of sensors per cluster $n_{\text {clust }}$ : This parameter specifies the number of sensors within each sensor cluster. For better separation performance, the number of sensors per cluster should be no less than the number of targets moving in the sensing range of a cluster. Potentially, all targets can move into the sensing range of one cluster; thus, we choose the number of sensors per cluster that is close to the number of targets in the field.

- Intercluster distance $d_{\text {inter }}$ : As shown in Fig. 4, the intercluster distance is the distance between two centers of neighboring sensor clusters. This parameter depends on $N$, i.e., the total number of sensors to be deployed in the field, and $n_{\text {clust }}$, i.e., the number of sensors per cluster.

- Intracluster distance $d_{\text {intra }}$ : Intracluster distance is the distance between the center of a sensor cluster and the farthest sensor within the same cluster. It is a measure of clustering degree. To avoid neighboring clusters merging, the parameter $d_{\text {intra }}$ should be less than $d_{\text {inter }} / 2$. Our initial experiments shown in Fig. 3 indicate that better separation performance is achieved when sensors are close to each other. However, it is not desired to cluster sensors in a very small area because 1) it may leave lots of 


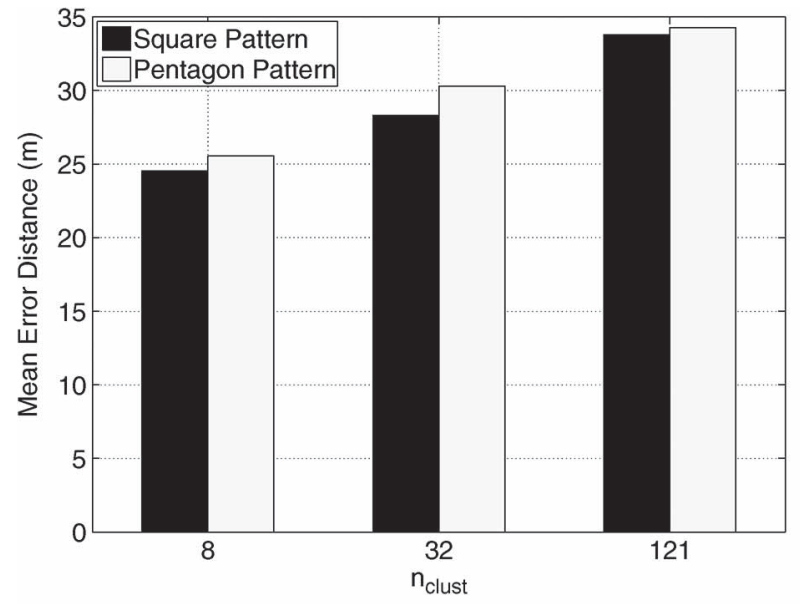

(a)

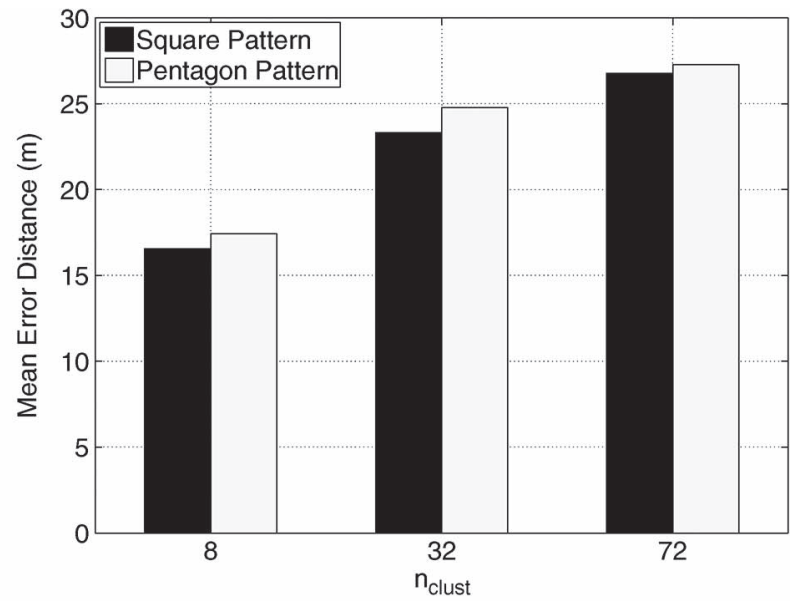

(b)

Fig. 5. Effect of in-cluster arrangement on tracking performance. (a) Error distance of $N=128$. (b) Error distance of $N=288$.

uncovered spots in the field when the sensor density is low. 2) When sensors are too close to each other, sensors receive roughly same aggregate signals. In this case, the separation performance cannot be good. Thus, we suggest $d_{\text {intra }}$ to be close to $d_{\text {inter }} / 4$. Our further experiments also support the choice of the parameter.

In addition to considerations on the separation performance, we propose cluster topologies because they satisfy the requirements listed in Section III-C: They are symmetric, and they can cover the whole field.

\section{Performance Evaluation}

Most existing research on target tracking with wireless sensor networks focuses on radio signals or audio signals. In this paper, we assume that audio signals are used for target tracking and that acoustic sensors are deployed in the field of interest. Readers interested in target tracking with radio signals may refer to the experiments in [3].

In this paper, we evaluate the proposed topologies with extensive simulations in MATLAB.

\section{A. Experiment Setup}

In the following experiments, the simulated field is a $1.6 \mathrm{~km} \times 1.6 \mathrm{~km}^{2}$ area. The movement of targets is restricted to a $1 \mathrm{~km} \times 1 \mathrm{~km}$ central area to eliminate boundary effects. The individual signals used for tracking are real bird signals downloaded from the website of Florida Museum of Natural History [30]. The aggregate signals received by sensors are simulated by first attenuating the individual sound signals according to the atmospheric sound absorption model [31] and then mixing the attenuated individual signals to form the aggregate signals. The BSS-based tracking algorithm is based on the aggregate signals only. We experiment with low-density sensor networks of density $N=128$ and 288 sensors. The sensing range of sensors is $250 \mathrm{~m}$.

In this paper, the BSS-based tracking algorithm works as follows. We use a BSS algorithm to recover individual signals from aggregate signals. Similar individual signals can be recovered from a number of sensors. The similarity is measured by the correlation between two recovered individual signals.
Clustering is used to find the recovered signal that is at the center of a cluster of similar recovered signals. The location is estimated by intersecting the sensing ranges of sensors that can receive similar signals. The intersection order is determined by the similarity to the center recovered signal, i.e., the correlation with the center recovered signal. A recovered signal with higher similarity will have the sensing range of the corresponding sensor be intersected earlier. The intersection ends before the intersected sensing range becomes zero.

We have tried a wide variety of BSS algorithms, including FastICA [32], Jade [33], and FPICA [21], for signal separation. Our experiments show that the difference in tracking performance caused by the choice of the BSS algorithms is very small for different topologies. In this paper, we use FastICA algorithm [32] for signal separation. FastICA is an efficient and popular algorithm for independent component analysis in terms of accuracy and low computational complexity. More details of the BSS-based tracking algorithm can be found in [3].

The performance metrics used in our experiments are the mean and the standard deviation of the error distance, as described in Section III-A. The experiment results presented below are averaged over 50 trials if not explicitly mentioned. In the following experiments, targets are moving at a speed below $0.15 \mathrm{~m}$ per sample interval. (In this paper, we focus on the effect of topologies on tracking performance. The effect of moving speed on tracking performance can be found in [25].) We compare the proposed cluster topology with the grid and random topologies in the experiments.

\section{B. Effect of In-Cluster Arrangement}

In this set of experiments, we investigate the effect of incluster topology. Here, we show experiments on the square and pentagon patterns as in-cluster arrangement. Similar experimental results are obtained from other polygons. Parameters used in this set of experiments are $d_{\text {intra }}=80 \mathrm{~m}$ and $n_{\text {targets }}=$ 10. As shown in Fig. 5(a) and (b), we observe that the tracking performance is not sensitive to the in-cluster arrangement since the difference between the two patterns is between $1.4 \%$ and $4.5 \%$ with different parameters. In the rest of the experiments, the sensors within a cluster are arranged into the square pattern. 


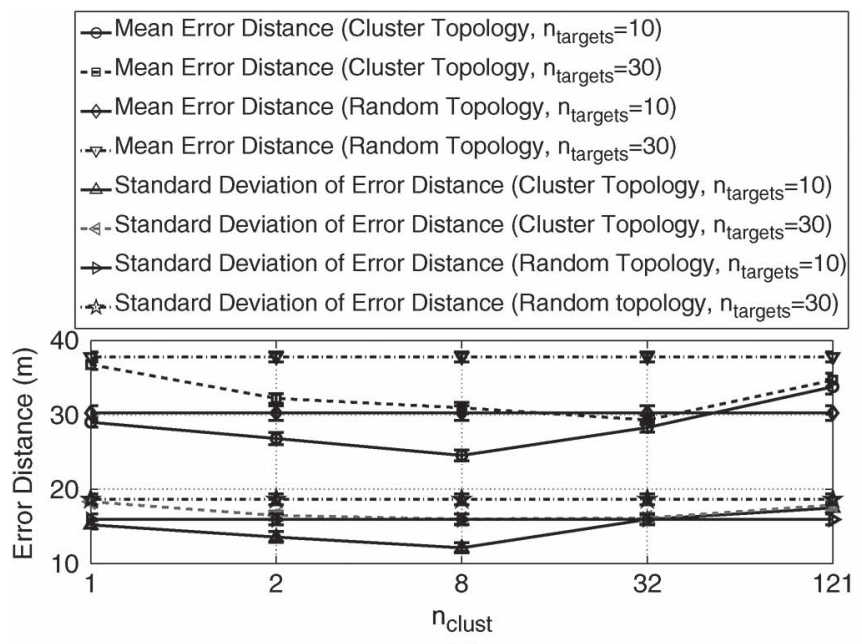

(a)

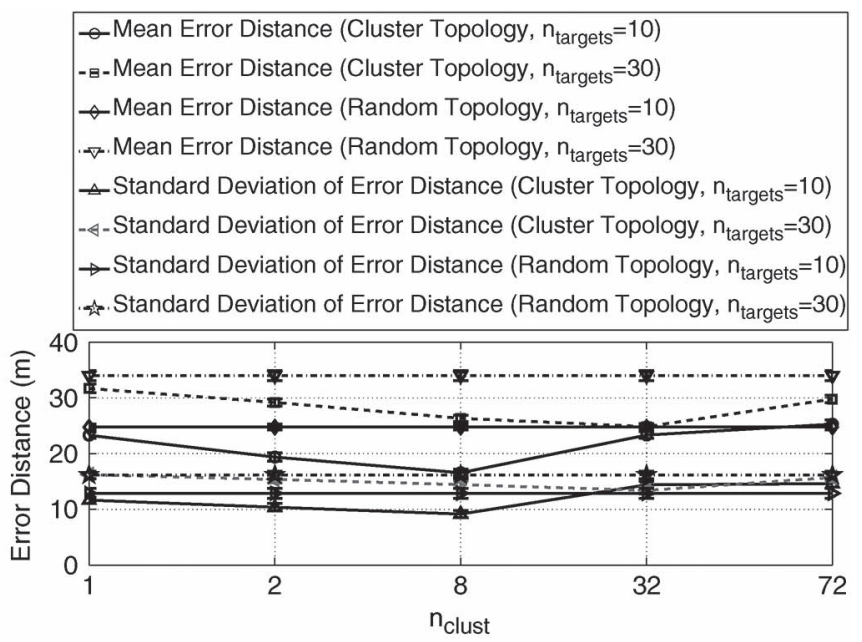

(b)

Fig. 6. Effect of number of sensors per cluster $\left(n_{\text {clust }}\right)$ with $95 \%$ confidence interval (When $n_{\text {clust }}=1$, the cluster topology essentially degenerates into the grid topology.) (a) $N=128$. (b) $N=288$.

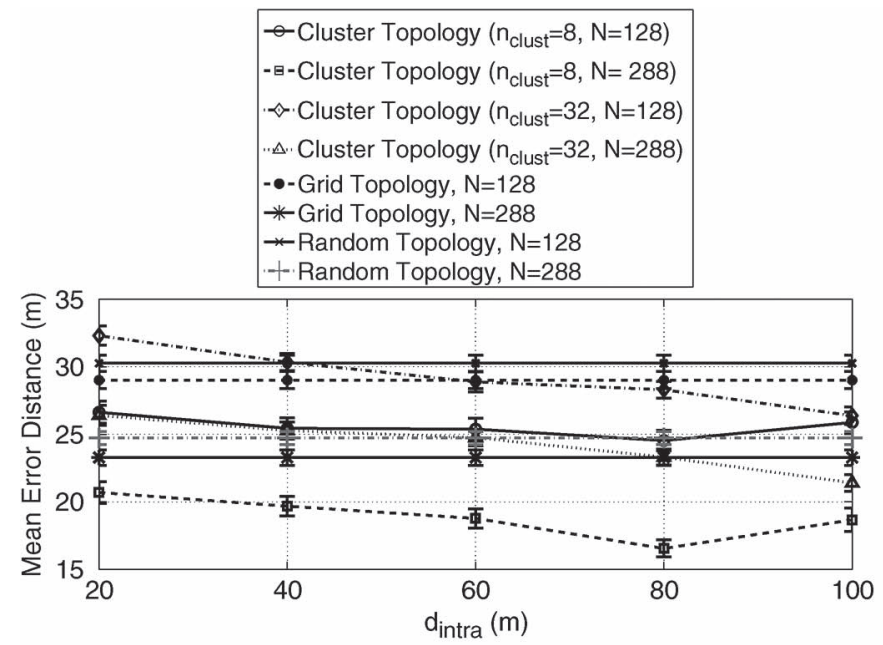

(a)

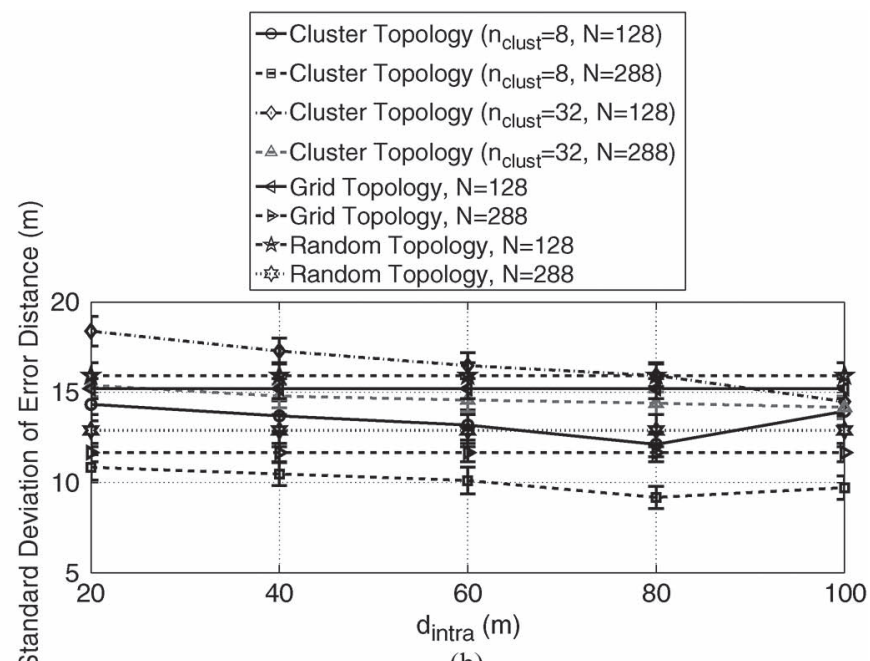

(b)

Fig. 7. Effect of intracluster distance $d_{\text {intra }}$ on tracking performance with $95 \%$ confidence interval. (a) Mean error distance. (b) Standard deviation of error distance.

\section{Number of Sensors per Cluster ( $n_{\text {clust }}$ )}

In this set of experiments, we focus on the number of sensors per cluster $n_{\text {clust }}$. The parameter $d_{\text {intra }}$ is set to be $80 \mathrm{~m}$ based on the conclusion drawn in Section IV-C. The intercluster distance depends on $N$, i.e., the sensor density, and $n_{\text {clust }}$, i.e., the number of sensors per cluster. When the sensor density is 128 (i.e., $N=128$ ), the intercluster distance $d_{\text {inter }}$ is 320 and 533.33 for $n_{\text {clust }}=8$ and $n_{\text {clust }}=32$, respectively. In addition, when $N=288$, the intercluster distance $d_{\text {inter }}$ is 228.5714 and 400 for $n_{\text {clust }}=8$ and $n_{\text {clust }}=32$, respectively. The number of moving targets $n_{\text {targets }}$ is 10 or 30 in this set of experiments. As shown in Fig. 6, in the case of 128 sensors $(N=128)$ and 10 targets $\left(n_{\text {targets }}=10\right)$, the minimum error distance is achieved when $n_{\text {clust }}=8$. In the case of 30 targets $\left(n_{\text {targets }}=30\right)$, the tracking performance is best when $n_{\text {clust }}$ is 32. This supports our conclusion made in Section IV-C. In comparison with the grid and random topologies, the performance increase of the cluster topology is $18.75 \%$ and $23.28 \%$ for 10 and 30 moving targets, respectively, when $N=128$.
When $N=288$, the increase is $26.12 \%$ and $27.90 \%$ for 10 and 30 moving targets, respectively. We can also observe that the performance of the cluster topology with selected parameters can be $33.62 \%$ and $32.78 \%$ better than the random and grid topologies, respectively.

\section{Effect of Intracluster Distance ( $\left.d_{\text {intra }}\right)$}

In this set of experiments, we investigate the effect of $d_{\text {intra }}$, i.e., the intracluster distance, on the tracking performance. The number of sensors per cluster $n_{\text {clust }}$ is set to be 8 or 32 , and the intracluster distance $d_{\text {intra }}$ varies from 20 to $100 \mathrm{~m}$. As shown in Fig. 7(a), the best tracking performance is achieved when $d_{\text {intra }}=80 \mathrm{~m}$. When the intracluster distance is very small or even close to zero, sensors within a cluster observe signals from the same set of targets. Thus, the aggregate signals received by the sensors within a cluster are close to each other. In turn, it degrades the separation performance. As shown in Fig. 7(a), the performance is getting better with the increase in 
TABLE I

Percentage Increase in Performance of Cluster Topology COMPARED WITH GRID AND RANDOM TOPOLOGIES

$$
\left(N=288, n_{\text {clust }}=32\right)
$$

\begin{tabular}{|c|l|l|}
\hline$n_{\text {targets }}$ & $\begin{array}{l}\text { Performance Increase over } \\
\text { Grid Topology }\end{array}$ & $\begin{array}{l}\text { Performance Increase over } \\
\text { Random Topology }\end{array}$ \\
\hline \hline 5 & 24.14 & 25.23 \\
10 & 25.87 & 27.45 \\
15 & 28.85 & 29.93 \\
20 & 30.22 & 30.94 \\
25 & 30.47 & 31.28 \\
30 & 32.78 & 33.62 \\
35 & 33.92 & 35.13 \\
40 & 35.02 & 37.18 \\
\hline
\end{tabular}

TABLE II

Percentage Increase in Performance of Cluster Topology COMPARED WITH GRID AND RANDOM TOPOLOGIES

$$
\left(N=128, n_{\text {clust }}=8\right)
$$

\begin{tabular}{|c|l|l|}
\hline$n_{\text {targets }}$ & $\begin{array}{l}\text { Performance Increase over } \\
\text { Grid Topology }\end{array}$ & $\begin{array}{l}\text { Performance Increase over } \\
\text { Random Topology }\end{array}$ \\
\hline \hline 5 & 17.23 & 22.59 \\
10 & 18.75 & 23.28 \\
15 & 19.54 & 23.64 \\
20 & 20.57 & 23.94 \\
25 & 21.12 & 25.55 \\
30 & 22.77 & 26.65 \\
35 & 24.37 & 27.48 \\
40 & 26.65 & 29.88 \\
\hline
\end{tabular}

$d_{\text {intra }}$. However, when the intracluster distance is larger than $80 \mathrm{~m}\left(d_{\text {intra }}>80 \mathrm{~m}\right)$, the error distance is increasing because the overlap of neighboring clusters' sensing ranges increases, and the bigger overlap area causes degradation in the tracking performance. The same trend can be observed on the standard deviation of the error distance, as shown in Fig. 7(b).

\section{E. Effect of Number of Targets $\left(n_{\text {targets }}\right)$}

In this set of experiments, we investigate the effect of the number of targets on the tracking performance. Table I shows the performance increase of the cluster topology over grid and random topologies averaged over 30 trials. The experiment parameters are as follows: density $N=288, n_{\text {clust }}=32$, and $d_{\text {intra }}=80 \mathrm{~m}$.

We can observe that the percentage of increase can achieve $37.18 \%$ and $35.02 \%$ for 40 targets over the random and grid topologies, respectively. Table I shows that performance increase becomes larger when the number of targets increases. It is mainly because better separation performance can be achieved for the cluster topology. Similar observations can be made in Tables II-IV, which shows the percentage of increase in performance of the cluster topology over the grid and random topologies with different experiment settings.

\section{F. Effect of High-Frequency Variations}

In this set of experiments, we experiment on the performance of tracking targets following paths with high-frequency variations. We focus on the path between two points with the physical distance of $300 \mathrm{~m}$ from each other. The path shape is sawtooth. In this set of experiments, we vary the sawtooth
TABLE III

Percentage Increase in Performance of Cluster Topology COMPARED WITH GRID AND RANDOM TOPOLOGIES

$$
\left(N=288, n_{\text {clust }}=8\right)
$$

\begin{tabular}{|c|l|l|}
\hline$n_{\text {targets }}$ & $\begin{array}{l}\text { Performance Increase over } \\
\text { Grid Topology }\end{array}$ & $\begin{array}{l}\text { Performance Increase over } \\
\text { Random Topology }\end{array}$ \\
\hline \hline 5 & 24.21 & 25.47 \\
10 & 26.12 & 27.90 \\
15 & 28.16 & 29.77 \\
20 & 29.52 & 29.86 \\
25 & 30.07 & 30.08 \\
30 & 32.66 & 33.02 \\
35 & 33.67 & 34.44 \\
40 & 34.87 & 36.53 \\
\hline
\end{tabular}

TABLE IV

Percentage Increase in Performance of Cluster Topology COMPARED WITH GRID AND RANDOM TOPOLOGIES $\left(N=128, n_{\text {clust }}=32\right)$

\begin{tabular}{|c|l|l|}
\hline$n_{\text {targets }}$ & $\begin{array}{l}\text { Performance Increase over } \\
\text { Grid Topology }\end{array}$ & $\begin{array}{l}\text { Performance Increase over } \\
\text { Random Topology }\end{array}$ \\
\hline \hline 5 & 16.83 & 22.29 \\
10 & 18.35 & 22.78 \\
15 & 18.64 & 23.94 \\
20 & 21.32 & 24.36 \\
25 & 22.47 & 26.25 \\
30 & 23.37 & 27.25 \\
35 & 25.27 & 27.98 \\
40 & 27.15 & 29.93 \\
\hline
\end{tabular}

frequency, i.e., the number of sawtooth periods, as shown in Fig. 8(a). When the frequency is equal to to zero, the path becomes a straight line path. As shown in Fig. 8(b), we can observe that mean error distance is increasing with the increase in frequency. The slight increase in the error distance with the number of sawtooth periods is because of higher speed required to finish longer paths. This experiments show the benefit of applying BSS algorithms in tracking targets: It enables tracking algorithms to have richer information for target tracking. Thus, the proposed algorithm can successfully track targets following paths with high-frequency variations even with low-density sensor networks in the cluster topologies.

\section{GUIDELINES FOR TOPOLOGY DESIGN}

In this paper, we focus on the topologies of sensor networks deployed for multitarget tracking with tracking algorithms based on the BSS technique. We propose the cluster topologies for BSS-based tracking algorithms. Our experiments indicate that better separation performance can be achieved when sensors are clustered together. In the proposed topologies, sensors are placed into clusters, and sensor clusters are evenly distributed in a field.

Based on the experiment results presented above, we summarize the guidelines for the topology design.

- To deploy a sensor network in a field of interest for tracking targets with BSS-based tracking algorithms, we propose cluster topologies. The clusters should be evenly distributed throughout the field.

- The number of sensors per cluster should be larger than the number of targets to be tracked within the field. 


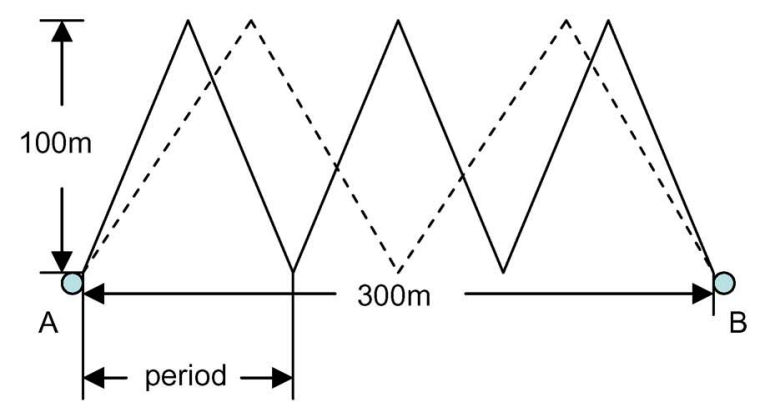

(a)

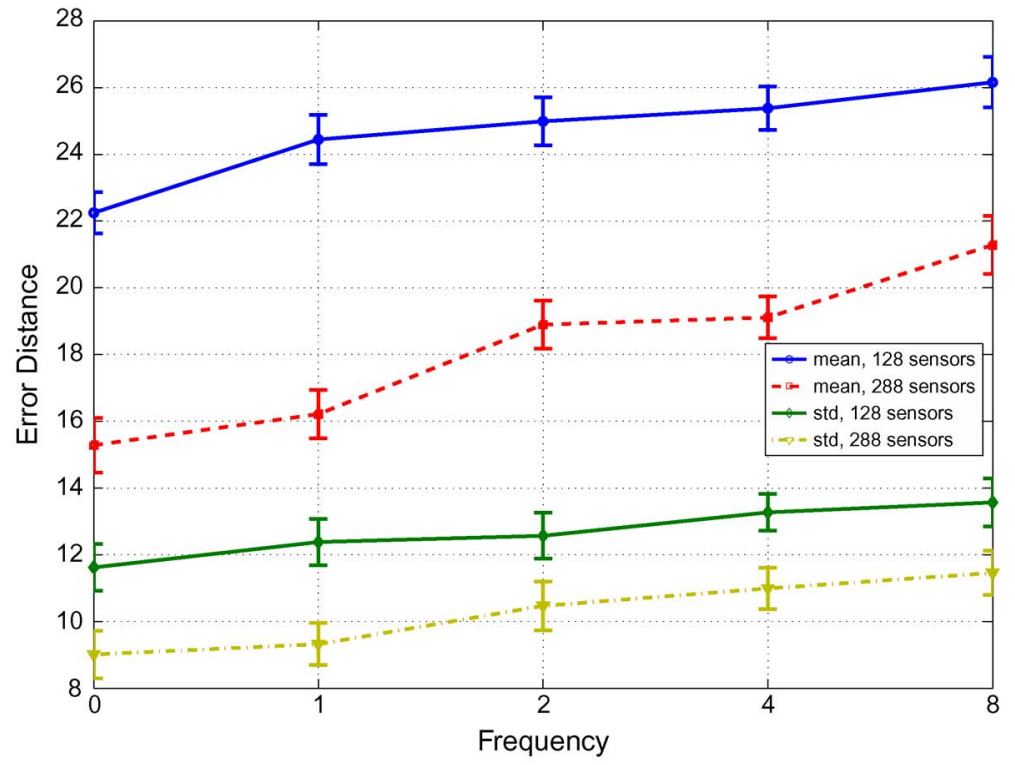

(b)

Fig. 8. Effect of target moving path on tracking performance with $95 \%$ confidence interval $\left(n_{\text {clust }}=8, d_{\text {intra }}=80\right.$, frequency: number of sawtooth periods). (a) Sawtooth paths. (b) Mean and standard deviation of the error distance.

- The tracking performance is not sensitive to the in-cluster arrangement; thus, the in-cluster arrangement can be in any polygon pattern.

- To cover the whole field, the intercluster distance $d_{\text {inter }}$ should be at least less than twice the sensing range of deployed sensors. Reducing the intercluster distance, i.e., increasing sensor density, can improve the tracking performance. The intracluster distance $d_{\text {intra }}$ should be close to $d_{\text {inter }} / 4$.

\section{CONCLUSION}

In this paper, we have proposed cluster topologies to improve tracking performance of BSS-based tracking algorithms. The topologies satisfy desired topology requirements: They are planned deployments with full coverage, and they are symmetrical. A set of guidelines on parameter selection for the proposed topologies is given in this paper. We evaluate the proposed topologies with extensive experiments. The proposed topologies can achieve more than 35\% improvement in tracking performance over the grid and random topologies. In conclusion, the proposed cluster topologies can significantly improve both the accuracy and the precision of the BSS-based tracking algorithm.

We plan to investigate the multipath effect in our future work. Currently, BSS-based tracking approaches [3] focus on tracking targets for outdoor applications or tracking in large indoor facilities such as stadiums. The approaches do not work well for indoor applications mainly because of the multipath effect.

\section{ACKNOWLEDGMENT}

Any opinions, findings, conclusions, and recommendations in this paper are those of the authors and do not necessarily reflect the views of the funding agency.

\section{REFERENCES}

[1] K. Pister, J. Kahn, and B. Boser, Smartdust: Wireless networks of millimeter-scale sensor nodes, Highlight Article in 1999 Electronics Research Laboratory Research Summary, 1999.

[2] J. Singh, U. Madhow, R. Kumar, S. Suri, and R. Cagley, "Tracking multiple targets using binary proximity sensors," in Proc. 6th Int. Conf. IPSN, 2007, pp. 529-538.

[3] Y. Zhu, A. B. Vikram, and H. Fu, "On tracking multiple indistinguishable targets," in Proc. 9th IEEE Int. Conf. MASS, Las Vegas, NV, USA, 2012, pp. 1-9.

[4] L. Tong, R. Liu, V. Soon, and Y.-F. Huang, "Indeterminacy and identifiability of blind identification," IEEE Trans. Circuits Syst., vol. 38, no. 5, pp. 499-509, May 1991.

[5] M. Ashourian, S. Woo, and H. Jeong, "Performance evaluation of blind source separation schemes for separating sensor signals in a distributed network," in Proc. Int. Conf. Convergence Inf. Technol., 2007, pp. 1884-1886.

[6] A. Hyvarinen, J. Karhunen, and E. Oja, Independent Component Analysis, 1st ed. Hoboken, NJ, USA: Wiley, May 2001. [Online]. Available: http://www.worldcat.org/isbn/047140540X

[7] N. Shrivastava, R. M. U. Madhow, and S. Suri, "Target tracking with binary proximity sensors: Fundamental limits, minimal descriptions, and algorithms," in Proc. 4th Int. Conf. SenSys, 2006, pp. 251-264. [Online]. Available: http://doi.acm.org/10.1145/1182807.1182833

[8] D. S. Friedlander and S. Phoha, "Semantic information fusion for coordinated signal processing in mobile sensor networks," Int. J. High Perform. Comput. Appl., vol. 16, no. 3, pp. 235-241, Aug. 2002.

[9] J. F. Scholl, J. R. Agre, and L. P. Clare, "Wavelet packet target classification schemes," in Proc. Meet. MSS Spec. Group Battlefield Acoust. Seismic Sens., 1999, pp. 79-95.

[10] X. Bai, S. Kumar, D. Xuan, Z. Yun, and T. H. Lai, "Deploying wireless sensors to achieve both coverage and connectivity," in Proc. 7th ACM Int. Symp. MobiHoc, 2006, pp. 131-142.

[11] H. Zhang and J. Hou, "Maintaining sensing coverage and connectivity in large sensor networks," Ad Hoc Sensor Wireless Netw., vol. 1, no. 1/2, pp. 89-124, 2005.

[12] R. Kershner, "The number of circles covering a set," Amer. J. Math., vol. 61, no. 3, pp. 665-671, Jul. 1939.

[13] Y. Zou and K. Chakrabarty, "Sensor deployment and target localization in distributed sensor networks," Trans. Embedded Comput. Syst., vol. 3, no. 1, pp. 61-91, Feb. 2004.

[14] H. Chan, M. Luk, and A. Perrig, "Using clustering information for sensor network localization," in Proc. 1st IEEE Int. Conf. DCOSS, 2005, pp. 109-125. [Online]. Available: http://dx.doi.org/10.1007/11502593_11

[15] T. Kasetkasem and P. K. Varsheny, "Communications structure planning for multisensor detection systems," Proc. Inst. Elect. Eng.-Radar, Sonar, Navigat., vol. 148, no. 1, pp. 2-8, Feb. 2001. 
[16] A. Alaybeyoglu, O. Dagdeviren, K. Erciyes, and A. Kantarci, "Performance evaluation of cluster-based target tracking protocols for wireless sensor networks," in Proc. 24th ISCIS, 2009, pp. 357-362.

[17] S. Bhatti, J. Xu, and M. Memon, "Clustering and fault tolerance for target tracking using wireless sensor networks," IET Wireless Sensor Syst., vol. 1, no. 2, pp. 66-73, Jun. 2011.

[18] J.-F. Cardoso, "Blind signal separation: Statistical principles," Proc. IEEE, vol. 86, no. 10, pp. 2009-2025, Oct. 1998.

[19] P. Comon, "Independent component analysis, a new concept?" Signal Process., vol. 36, no. 3, pp. 287-314, Apr. 1994.

[20] Z. He, L. Yang, J. Liu, Z. Lu, C. He, and Y. Shi, "Blind source separation using clustering-based multivariate density estimation algorithm," IEEE Trans. Signal Process., vol. 48, no. 2, pp. 575-579, Feb. 2000.

[21] A. Hyvarinen, "Fast and robust fixed-point algorithms for independent component analysis," IEEE Trans. Neural Netw., vol. 10, no. 3, pp. 626634, May 1999.

[22] A. Hyvrinen and E. Oja, "A fast fixed-point algorithm for independent component analysis," Neural Comput., vol. 9, no. 7, pp. 1483-1492, Oct. 1997.

[23] M. Gaeta and J.-L. Lacoume, "Source separation without a priori knowledge: The maximum likelihood solution," in Proc. EUSIPCO, 1990, pp. 621-624.

[24] D.-T. Pham, P. Garrat, and C. Jutten, "Separation of a mixture of independent sources through a maximum likelihood approach," in Proc. EUSIPCO, Brussels, Belgium, Aug. 1992, pp. 771-774.

[25] A. Vikram, "Tracking in wireless sensor network using blind source separation algorithms," M.S. thesis, Cleveland State Univ., Cleveland, OH, USA, 2010.

[26] W. Kim, K. Mechitov, J.-Y. Choi, and S. Ham, "On target tracking with binary proximity sensors," in Proc. 4th Int. Symp. IPSN, 2005, pp. 301-308.

[27] T. He, P. Vicaire, T. Yan, L. Luo, L. Gu, G. Zhou, R. Stoleru, Q. Cao, J. A. Stankovic, and T. Abdelzaher, "Achieving real-time target tracking using wireless sensor networks," in Proc. 12th IEEE RTAS, 2006, pp. 37-48.

[28] A. Hyvärinen and M. Inki, "Estimating overcomplete independent component bases for image windows," J. Math. Imag. Vis., vol. 17, no. 2, pp. 139-152, Sep. 2002. [Online]. Available: http://dx.doi.org/10.1023/A: 1020633503820

[29] A. Hyvarinen, R. Cristescu, and E. Oja, "A fast algorithm for estimating overcomplete ICA bases for image windows," in Proc. IJCNN, 1999, vol. 2, pp. 894-899.

[30] J. W. Hardy, Sounds of Florida's Birds, 1998. [Online]. Available: www. flmnh.ufl.edu/birds/sounds.htm

[31] L. E. Kinsler, A. R. Frey, A. B. Coppens, and J. V. Sanders, Fundamentals of Acoustics. New York, NY, USA: Wiley, 2000.

[32] C. Hesse and C. James, "The fastica algorithm with spatial constraints," IEEE Signal Process. Lett., vol. 12, no. 11, pp. 792-795, Nov. 2005.

[33] J. F. Cardoso and A. Souloumiac, "Blind beamforming for non Gaussian signals," Proc. Inst. Elect. Eng. F-Radar Signal Process., vol. 140, no. 6, pp. 362-370, Dec. 1993. 